\title{
Publisher's Note: Tomonaga-Luttinger liquid and localization in Weyl semimetals [Phys. Rev. B 95, 205143 (2017)]
}

Xiao-Xiao Zhang and Naoto Nagaosa

(Received 6 June 2017; published 12 June 2017)

DOI: 10.1103/PhysRevB.95.239904

This paper was published online on 31 May 2017 with a production error that affects the first sentence of the Acknowledgments on page 5. The first sentence of the Acknowledgments has been deleted as of 6 June 2017. The Acknowledgments are correct in the printed version of the journal. 